\title{
Effects of High Fructose/Glucose on Nlrp3/I11ß Inflammatory Pathway
}

\author{
Erinola Araoye $^{1^{*}}$, Karina Ckless ${ }^{1}$
}

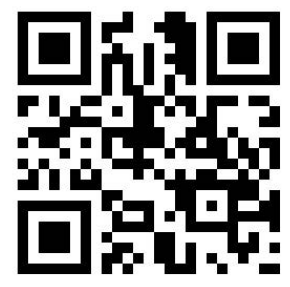

The Nod-Like Receptor Protein-3/Interleukin-1 $\beta$ (NLRP3/IL-1 $\beta$ ) inflammatory pathway activation is associated with autoimmune diseases including gout, Muckle-Wells syndrome, familial Mediterranean fever and multiple sclerosis. Type 2 diabetes and insulin resistance have been associated with chronic inflammation; however, the mechanism by which the NLRP3 inflammatory pathway participates in this condition remains unclear. Past research shows that fructose could induce production of reactive oxygen species (ROS), which are involved in NLRP3 complex assembly and secretion of IL-1 $\beta$. In this study, the activation of NLRP3 inflammatory pathway was investigated in mouse macrophage cells (J774A1) treated with fructose and glucose $(25,50$ and $100 \mathrm{mM})$ for 24 hours. The cell lysates were analyzed using western blot analysis for inflammatory protein components NLRP3, IL-1 $\beta$, and caspase 1 , as well as antioxidant enzymes including peroxiredoxins ( $\left.\mathrm{PRXSO}_{3}, \mathrm{PRX} 1\right)$, superoxide dismutase (SOD) and catalase. Mitochondria-derived ROS and mitochondrial permeability were assessed by MitoSOX red staining and JC-1 assay respectively. Results suggest that the intracellular levels of pro-IL-1 $\beta$, antioxidant proteins (PRXSO3, SOD) and ROS were more elevated in cells treated with fructose and glucose at 25,50 and $100 \mathrm{mM}$ when compared to untreated cells. Although pro-IL-1 $\beta$ accumulation was observed, no extracellular IL-1 $\beta$ could be detected using enzyme-linked immunosorbent assay (ELISA).

\section{INTRODUCTION}

Western culture has adopted a diet rich in energy-loaded carbohydrates. This increased consumption of high-energy foods has been accompanied by reliance on mechanical technology to do work, reducing necessary physical activity (Popkin, 2001). The ratio of energy consumed to energy spent is imbalanced in favor of consumption, which results in storage of fat cells as adipose tissue and uncontrolled deposition of fats could lead to an individual carrying an excess amount of weight, referred to as being overweight or obese. This condition can be defined using the body mass index (BMI) of an individual (Finucane et al, 2011). Higher BMIs correspond to excess weight and obesity. Studies (Finucane et al, 2011) show that the mean BMI worldwide has increased over the years and so has the rate of obesity. In 2008, over 500 million people worldwide were considered obese and about 1.46 billion were overweight (Finucane et al, 2011).

In obese individuals, enlarged fat cells secrete fatty acids and cytokine factors such as tumor necrosis factor- $\alpha$ (TNF- $\alpha$ ), that are capable of causing a wide range of downstream effects such as having higher risks for a variety of diseases including coronary

${ }^{1} 101$ Broad street, Plattsburgh, New York. SUNY Plattsburgh Chemistry Dept.

*To whom correspondence should be addressed: earaoye@yahoo.com

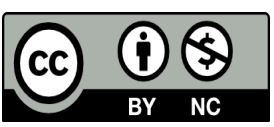

Except where otherwise noted, this work is licensed under https://creativecommons.org/licenses/by-nc/4.0/ doi: 10.22186/jyi.31.5.25-30. artery disease, hypertension, metabolic syndrome, gall bladder disease, cancer, osteoarthritis and type 2 diabetes (Rodriguez-Hernandez, Simental-Mendia, Rodriguez-Ramirez, \& Reyes-Romero, 2013). Among the obesity related diseases, type 2 diabetes has recently been classified an autoimmune disease involving inflammation through NLRP3 activation (Bray, 2004; Gunter \& Leitzmann, 2006; Hajer, Haeften, \& Visseren 2008). Studies have investigated the relationship between type 2 diabetes, insulin resistance and IL$1 \beta$ expression (Gao et al, 2014; Larsen et al, 2007). Larsen et al.'s experimental results (2007) showed that blockade of IL-1 $\beta$ expression in patients with type 2 diabetes improved $\beta$-cell function and promoted glycemic control while Goa et al.'s results (2014) showed that IL-1 $\beta$ presence in human adipocytes significantly reduced the gene expression of insulin signaling molecules and its absence improved insulin sensitivity. The secretion of IL-1 $\beta$ is regulated by the Nod-Like Receptor protein 3 (NLRP3) inflammasome. IL-1 $\beta$ secretion is carried out in two steps. The first signal, also known as the priming step, consists of activation of Nod-Like Receptor protein 3 (NLRP3) coupled with accumulation of pro- IL-1 $\beta$ - the inactivated precursor protein for IL-1 $\beta$. Upon accumulation of the precursor, a second signal is needed to recruit the NLRP3 inflammasome complex, consisting of (NLRP3), adaptor protein apoptosis speck-like Protein (ASC) and activated caspase 1, consequently responsible for cleavage of pro-IL-1 $\beta$ to secretion as Il-1 $\beta$ (Figure 1). When NLRP3/IL-1 $\beta$ pathway is activated, ROS production is also observed (Jo, Kim, Shin, \& Sasakawa et al, 2016). An article (Tschopp \& Schroder, 2010) suggested that mitochondrial ROS is not only correlated with NLRP3 activation, but is involved in assembling the NLRP3 inflammasome complex.

Mitochondria are considered the main source of ROS in 


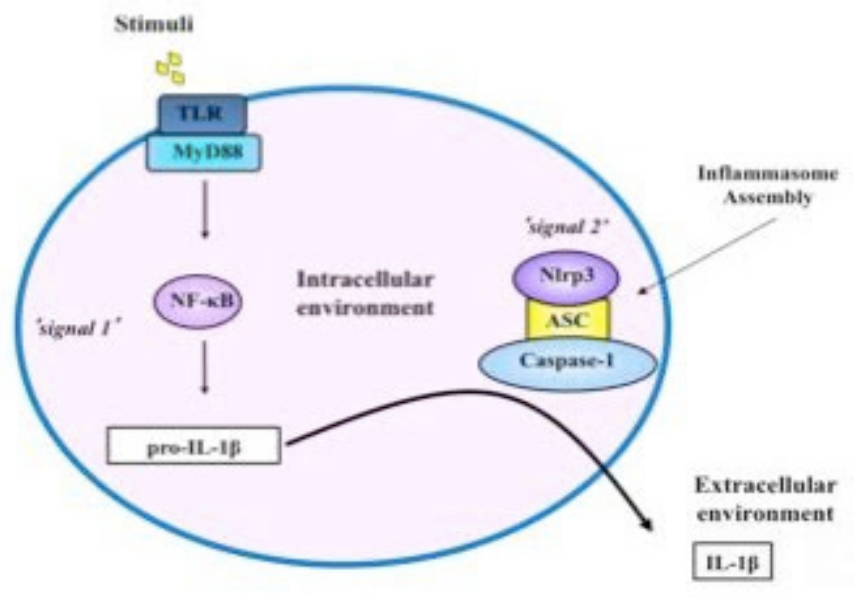

Figure 1. Diagram summarizing the NLRP3 inflammasome activation and IL-1ß secretion. Signal 1 is the priming step, where inactive precursor, pro- IL-1 $\beta$, is accumulated. Signal 2 involves the assembly of an inflammasome complex consisting of NLRP3, ASC and Caspase-1, which is responsible for cleavage of pro-IL-1 $\beta$ into the active form IL-1 $\beta$, for further secretion by the cells.

normal and altered metabolism, and are the site of aerobic carbohydrate metabolism (Lenaz, 2001). After consumption, carbohydrates are broken down to sugars, which are metabolized to yield acetyl-CoA, the primary substrate of the tricarboxylic acid cycle (TCA) taking place in the mitochondria. During the TCA cycle, Acetyl-CoA goes though series of oxidation steps carried out by co-enzymes $\mathrm{NAD}^{+}$and FAD, which are reduced to NADH and $\mathrm{FADH}_{2}$ respectively. These energy rich hydrogen atoms are supplied to the electron transport chain (ETC), where they are reoxidized simultaneously with the reduction of oxygen to water by complexes on the mitochondrial membrane, yielding ATP (adenosine triphosphate), the primary form of energy in cells. This process results in $1-2 \%$ of consumed oxygen being partially reduced to generate superoxide anion $\left(\mathrm{O}^{\bullet}\right)$ (Thannickal \& Fanburgh, 2000). Antioxidants usually act collectively to suppress ROS and other free radicals by reducing them to a less reactive species as in the multistep reduction of superoxide to water. Superoxide dismutase (SOD) reduces $\mathrm{O}^{\bullet-}$ to hydrogen peroxide $\left(\mathrm{H}_{2} \mathrm{O}_{2}\right)$, which is further reduced by catalase (CAT) or Glutathione peroxidase (GPX) to water (Devasagayam et al., 2004). Peroxiredoxins (PRX) are able to directly reduce $\mathrm{H}_{2} \mathrm{O}_{2}$ to water, while GPX works to reduce $\mathrm{H}_{2} \mathrm{O}_{2}$ by using Glutathione (GSH), a thiol-based antioxidant, as a substrate. In an adverse physiological state referred to as oxidative stress, the body is overwhelmed with production of ROS resulting in an imbalance of ROS/antioxidant ratio (Nordberg \& Arner 2001). Oxidative stress has been associated with several pathological conditions such as cancer, diabetes (Ceriello \& Motz, 2004) and chronic inflammation as well as other autoimmune diseases (Reuter et al, 2010). Among the inflammatory pathways that are associated with chronic inflammation and autoimmune diseases is the NLRP3 Inflammasome/IL-1 $\beta$ Inflammatory pathway (Jo et al,
2016).

ROS direct involvement with NLRP3 inflammasome activation has not been completely elucidated. The purpose of this study was to investigate whether or not ROS production is proportional to the sugar concentration in macrophage cell culture and if this higher input of glucose and fructose would contribute to an inflammatory response through the NLRP3 inflammasome. Our findings will give insight on the impact of a sugar-rich diet on oxidative and inflammatory pathways, as well as provide a perspective on macrophage cellular processes involved with obesity-associated inflammation for other scientists and researchers to expand on.

\section{MATERIALS AND METHODS \\ Chemicals and Reagents}

3, 3', 5, 5'-tetramethylbenzidine (TMB) reagent, Antimycin A (AA) and $\beta$-actin antibody were obtained from Sigma. Mitochondrial superoxide indicator (MitoSOX red, was purchased from Molecular Probes ${ }^{\circledR}$ (InvitrogenTM). NLRP3 antibody, caspase 1 antibody and IL-1 $\beta$ antibody was obtained from Santa Cruz. PRX1 and $\mathrm{PRXSO}_{3}$ antibodies were obtained from Abcam. The secondary IgG peroxidase-linked mouse antibody, and rabbit antibodies were purchased from GE Healthcare. All reagents were of analytical grade.

\section{Cell Culture and Experimental Conditions}

Mouse monocyte (macrophage) cell line J774A1 was obtained from American type culture collection (ATCC). Cells were seeded at $1 \times 10^{5}$ cells $/ \mathrm{mL}$ using RPMI 1640 medium supplemented with $10 \% \mathrm{FBS}$, penicillin, streptomycin and L-glutamine, and incubated at $37^{\circ} \mathrm{C}$ in a $5 \% \mathrm{CO} 2$-supplemented atmosphere for at least 24 hours before treatments. The cells were treated with glucose or fructose at concentrations $25 \mathrm{mM}, 50 \mathrm{mM}$ and $100 \mathrm{mM}$ for 24 hours. Treatments were performed in triplicates.

\section{Cell Harvesting and Lysates Preparation}

After respective treatments, tissue culture plates were placed on ice and the attached cells were rinsed once with cold phosphate buffered saline (PBS) and lysed using total lysis buffer $(50 \mathrm{mM}$ Tris, pH 7.4, 150mM Sodium Chloride ( $\mathrm{NaCl}), 2 \mathrm{mM}$ ethylenediaminetetraacetic acid (EDTA), $0.2 \%$ Triton $^{\mathrm{TM}} \mathrm{X}-100,0.3 \%$ IGEPAL ${ }^{\circledR}$, and protease inhibitor cocktail). Lysates were removed from the plate, transferred to microcentrifuge tubes, and immediately frozen in liquid nitrogen to prevent protein degradation and enhance cell lysis.

\section{Protein Concentration Determination: Bradford assay}

The J774A1 lysates were thawed at room temperature. $10 \mu \mathrm{L}$ of bovine serum albumin (BSA) standards or cell lysates samples were transferred into separate clean tubes and $1000 \mu \mathrm{L}$ of the 1:4 diluted dye (Bradford) was added to the respective tubes. The tubes were incubated for five minutes at room temperature before their content was transferred into a cuvette. Absorbance was measured at $595 \mathrm{~nm}$ and the protein content in the samples was calculated using a standard curve with a series of BSA standards to $1500-100 \mathrm{mg} /$ 
$\mathrm{ml}$. The amount of protein in each condition was determined and adjusted to $0.5 \mathrm{mg} / \mathrm{ml}$ by adding PBS buffer.

\section{Sodium Dodecyl Sulfate Polyacrylamide Gel Electrophoresis (SDS-PAGE) and Immunoblot}

Leamli SDS buffer was added to the lysate and $30 \mu \mathrm{L}$ of the mixture per condition was loaded on to polyacrylamide gels. The proteins were separated on $10 \%$ SDS-PAGE and transferred from the gel to a nitrocellulose membrane. The membrane was blocked for one hour at RT with $3 \%$ milk in PBS containing $0.05 \%$ Tween ${ }^{\circledR 2} 20$ (PBST) and then incubated with respective primary antibodies overnight at $4^{\circ} \mathrm{C}$ on a rotating platform. The membranes were washed three times with PBST for five minutes each and incubated with respective horseradish peroxidase-conjugated secondary antibodies. Western blots were developed utilizing, 3, 3', 5, 5' TMB liquid substrate system for membranes according manufacturer's instructions.

\section{Detection of Mitochondria-Derived ROS}

Mitochondria-derived ROS, was detected using the mitochondrial superoxide indicator, MitoSOX red, a cationic dihydroethidium modified to target the mitochondria. According to the manufacturer, MitoSOX red is a cell-permeable dye that reacts with ROS to form ethidium, which upon binding to nucleic acids gives a bright red fluorescence. Briefly, cells were seeded onto eight well CultureSlides (BD Falcon ${ }^{\mathrm{TM}}$ ) and treated as described above. At the end of the respective treatments, the cells were rinsed with PBS and loaded with MitoSOX red $(2.5 \mu \mathrm{M})$ for ten minutes. The medium containing fluorescent probes was removed, and the cells were rinsed with PBS, and observed in an Olympus BX53 Fluorescence Microscope coupled to an Olympus DP73 digital camera.

\section{Detection of Mitochondrial Membrane Potential ( $\Delta \psi \mathrm{m})$}

Cells $(5 \times 105$ cells $/ \mathrm{mL})$ were seeded in 12-well plates and 24 hours later were treated as indicated. After washing with PBS, cells were incubated in fresh medium containing 5,5',6,6'-tetrachloro1,1',3,3'-tetraethylbenzimidazol-carbocyanine iodide (JC-1) for $15 \mathrm{~min}$. The dye was then removed; and cells were washed with PBS and fresh medium was added. Then live cells were immediately observed in an Olympus BX53 Fluorescence Microscope coupled to Olympus DP73 digital camera. Healthy cells, mainly JC-1 aggregates were observed at 540/570nm excitation/emission and the apoptotic or unhealthy cells with mainly JC-1 monomers at $485 / 535 \mathrm{~nm}$ excitation/emission.

\section{RESULTS}

\section{Mitochondria-derived ROS}

As ROS are a by-product of fructose and glucose metabolism, it was anticipated that an increase in substrate would cause an increase in ROS production. This increase in ROS could promote an environment suitable for NLRP3 inflammasome activation. In the vicinity of ROS, MitoSOX red produces a bright red fluorescence, which varies in intensity with amount of ROS present. Antimycin A was used as the positive control as it is known to be an inhibitor of complex III in the ETC and leads to robust ROS production and mitochondrial damage. J774A1 cells appeared to produce more red fluorescence when treated with fructose and glucose in contrast to the control, untreated cells (Figure 2a). In addition, fructose treatment elicits a more intense fluorescence than glucose at concentrations 25 and $50 \mathrm{mM}$, as demonstrated by fluorescence intensity analysis (Figure 2b).

\section{Mitochondrial Membrane Potential $(\Delta \psi \mathrm{m})$}

When the mitochondrion is constantly hyperactive, it has the tendency to go into a dysfunctional state evidenced by leaks in the mitochondrial membrane. These leaks could cause it to become depolarized (Tsujimoto \& Shimizu, 2007). The JC-1 dye kit can be used to detect the overall health of the mitochondria. In hyperpolarized mitochondria the dye emits a red fluorescence while in depolarized mitochondria it emits green. Hence, the red fluorescence indicates healthier mitochondria while green indicates a disturbed mitochondrial function. In cells treated with glucose $(25 \mathrm{mM})$ and fructose $(25 \mathrm{mM}, 50 \mathrm{mM})$, there is a higher red intensity compared with control, indicating that the mitochondrion is polarized. However, as treatment concentration is increased, there is a decrease in red and increase in green fluorescence in glucose treatment, indicating mitochondrial depolarization occurs. It appears that the decrease in red and increase in green was more intense in fructose treated cells than glucose treated cells at $100 \mathrm{mM}$ concentration (Figure 3a \& b).

\section{Western Blot analysis for Antioxidant and Inflammatory Pro-} teins

Western blot analysis was used to detect the presence of antioxidant and inflammatory proteins. Comparing the intensity and thickness of the bands can give insight on the amounts of proteins present across experimental conditions. The Bradford assay was used to measure the total amounts of protein in each sample and ensure the total protein loaded on to gels were constant throughout the treatment conditions. Increased expression of antioxidant protein is a marker of oxidative stress. As a response to counteract ROS, antioxidant enzymes are produced through stress response mechanisms to prevent oxidative damage (Nguyen et al, 2009). Compared with the control experiment, PRX1 levels did not seem to vary throughout the conditions, however, as implied by the prominent bands observed, the amounts of its oxidized form (PRXSO3) and catalase appear to be increased, particularly with fructose treatment. SOD1 expression showed to be slightly increased in both fructose and glucose treatments (Figure 4).

The presence and amount of precursor proteins of NLRP3 inflammatory pathway can be used to assess NLRP3 inflammasome activation and determine the extent of inflammatory response in macrophage cells. The precursor protein, Pro-IL-1 $\beta$, appeared to be increased only in fructose and glucose-treated cells. In addition, it seems to be expressed in higher amounts in fructose treatments compared to glucose treatments. The cleaved products of caspase 1 , seen as the multiple lower bands, are also observed majorly in fructose condition, indicating some level of caspase 1 activation. NLRP3 protein, however, seems to remain constant throughout the conditions (Figure 5). 


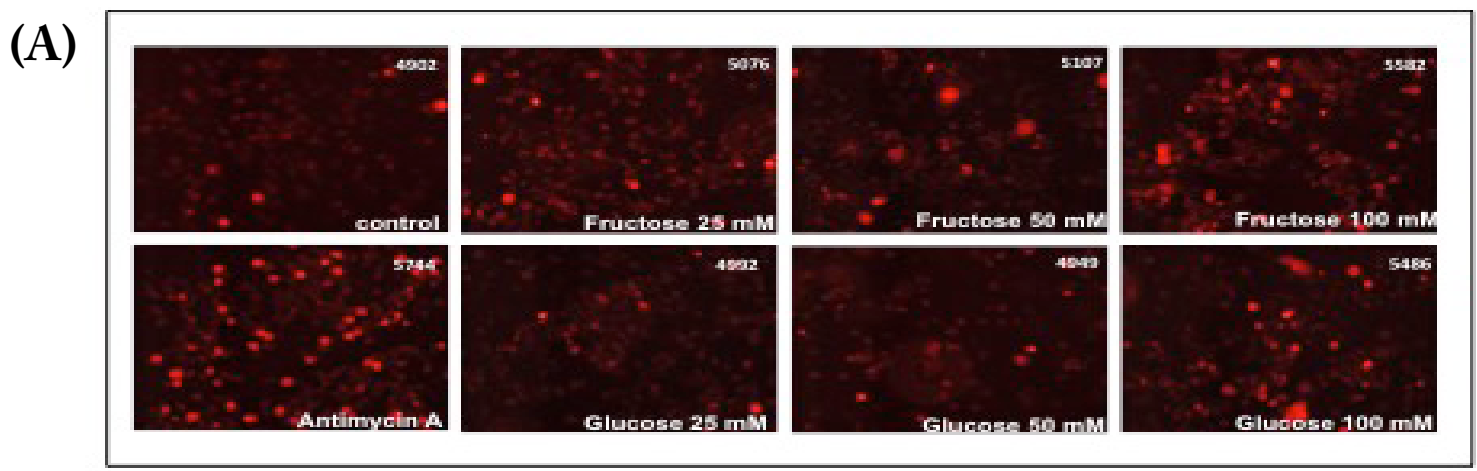

(B)

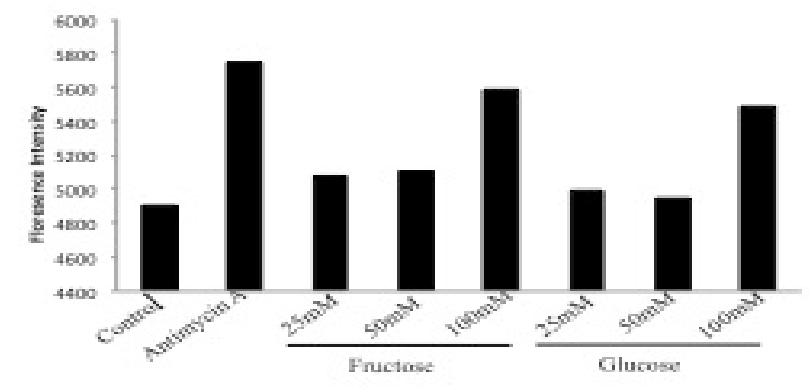

Figure 2. Mitochondria-derived ROS. J774A1 cells were treated with increased concentrations, $25 \mathrm{mM}, 50 \mathrm{mM}$ and $100 \mathrm{mM}$, of fructose or glucose for 24h, and then incubated for ten minutes with MitoSOX red. Images (Figure 2a) were taken using Olympus BX53 fluorescence microscope. Amount of mitochondrial ROS was indicated by intensity of red fluorescence. The numbers on the top right of each panel indicate the fluorescence intensity (arbitrary units) analyzed by ImageJ image analysis software. The bar chart below (Figure 2b) depicts the fluorescence intensities as shown in the panels.

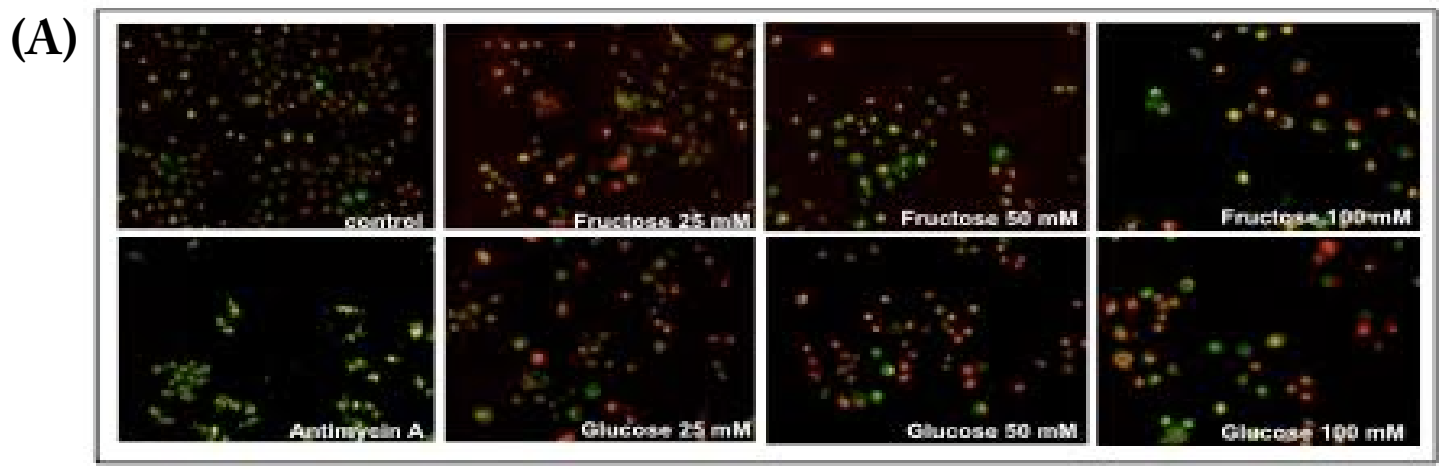

(B)

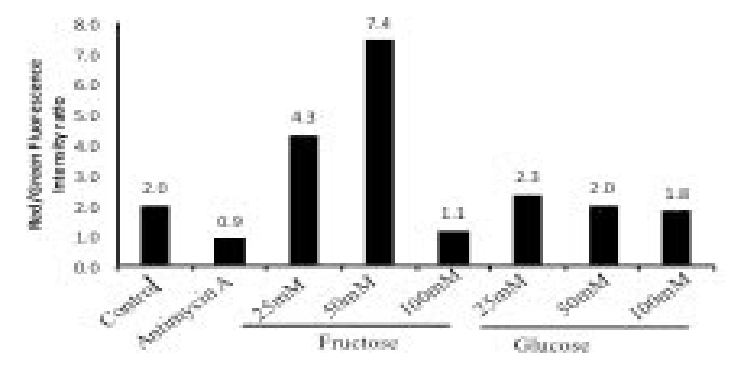

Figure 3: Mitochondrial permeability. J774A1 cells were treated with increased concentrations, 25mM, 50mM and 100mM, of fructose or glucose for 24h, and then incubated with JC-1 dye for 15 minutes. The pictures (Figure 3a) of live cells were taken using the Olympus BX53 fluorescence microscope at 540/570 nm (red) for healthy cells and 485/535 nm (green) for unhealthy cells. An increase in red/green fluorescence intensity indicates healthy or hyperpolarized mitochondria, illustrated on the graph on the bottom (Figure 3b). The red/green ratio was calculated using integrated intensity of red and green channels measured by ImageJ software. 


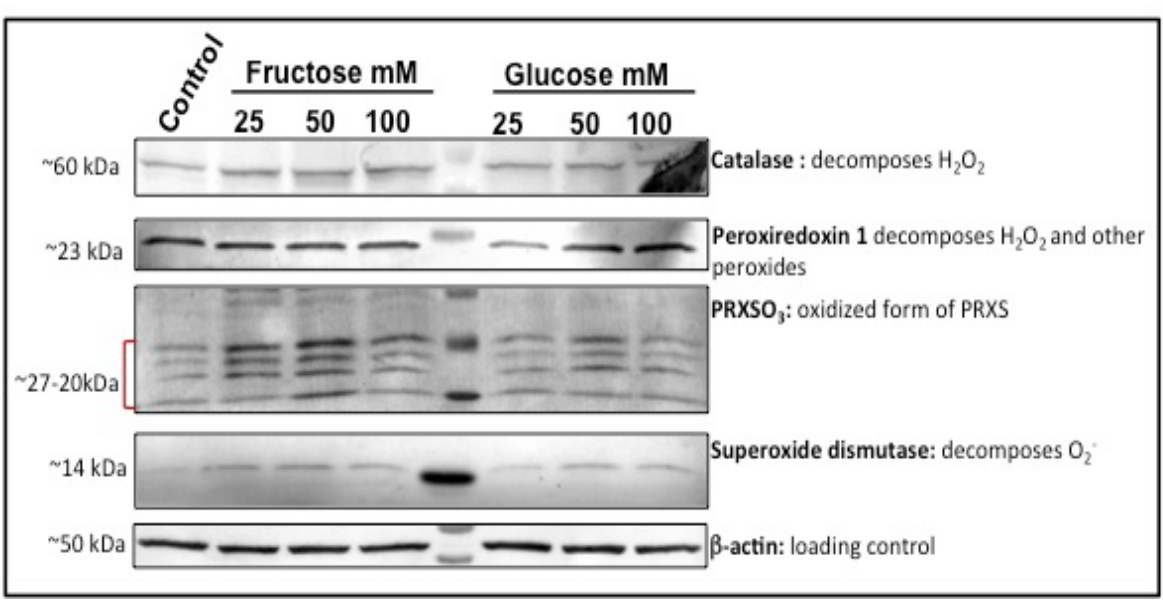

Figure 4: Antioxidant enzymes. J774A1 cells were treated with fructose or glucose at 25mM, $50 \mathrm{mM}$ and $100 \mathrm{mM}$ for $24 \mathrm{~h}$ after which they were lysed using lysis buffer. Total amount of antioxidant enzymes, catalase $(60 \mathrm{KDa})$, peroxiredoxin 1 (PRX1,23KDa), the oxidized from of peroxiredoxins (PRXSO3) ranging from 27-20KDa and superoxide dismutase 1 (SOD1, $14 \mathrm{KDa})$, were analyzed by western blotting. $\beta$-actin $(50 \mathrm{KDa})$ was used as loading control for total protein content.

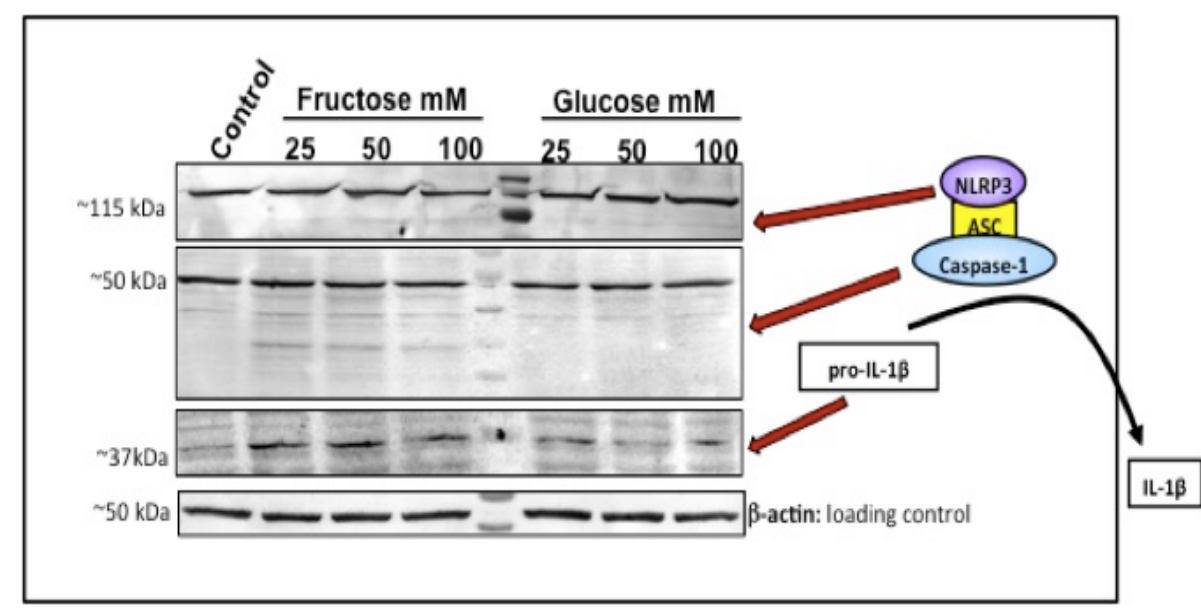

Figure 5: NLRP3 inflammasome complex proteins. J774A1 cells were treated with fructose or glucose at $25 \mathrm{mM}, 50 \mathrm{mM}$ and $100 \mathrm{mM}$ for $24 \mathrm{~h}$ after which they were lysed using lysis buffer. Total amount of intracellular components of the NLRP3 inflammasome complex proteins, NLRP3 $(115 \mathrm{KDa})$, caspase $1(50 \mathrm{KDa})$ and pro-1L-1 $\beta$ (37KDa) were detected by western blotting. $\beta$ - actin $(50 \mathrm{KDa})$ was used as loading control for total protein content.

The mitochondrial permeability assay suggests that the lower concentrations of fructose $(25 \mathrm{mM}, 50 \mathrm{mM})$ and glucose $(25 \mathrm{mM})$ treatments actually cause the cells to become relatively healthy compared to the control. However, as the treatment concentrations increased, the membrane was depolarized, indicating an unhealthy cell. One of the key events during apoptosis and necrosis, or cell death, is an increase in mitochondrial membrane permeability by opening of permeability transition pore proteins, which are ion channels. This leak in the membrane leads to a decrease in membrane potential of the mitochondria (Tsujimoto \& Shimizu, 2007). Another study (Russell et al., 2002) also found a dose-dependent increase in ROS production and mitochondria depolarization in neurons treated with glucose. This study (Russell et al., 2002) observed that the mitochondrial membrane goes through hyperpolarization before it is depolarized suggesting that depolarization might be time dependent. Future studies could investigate this by measuring membrane potential at varied time points.

As ROS levels appeared to increase with treatment, the intracellular antioxidant protein amounts were investigated. It appeared that the amounts of some antioxidant proteins (Catalase and $\mathrm{PRXSO}_{3}$ ) were increased in treated cells, indicating an upregulation of antioxidant protein expression and implying an increased level of oxidative stress. We found that fructose had a more observable effect on this response. This could be due to the differences in fructose and glucose metabolism. According to Cha et al (2008) glucose metabolism is more regulated than that of fructose. In the glycolytic pathway, fructose is able to bypass the rate-limiting step catalyzed by phosphofructokinase, an important control enzyme for the rate of glucose metabolism, allowing fructose to be metabolized at a faster rate. In fructose fed mice, it was found that fructose metabolic pathway, and not glucose, was essential to recruitment of macro-

\section{DISCUSSION AND CONCLUSIONS}

The results appear to be consistent with the presumption that sugars can induce oxidative stress through an over active mitochondria and promote an inflammatory response in J774A1 macrophage cells. The MitoSOX red assay possibly indicates an increased ROS production in treated cells. In lower concentrations $(25 \mathrm{mM}, 50 \mathrm{mM})$, fructose seems to invoke more ROS production than glucose, however in the highest concentration, fructose and glucose treatments produced comparable amounts of ROS, perhaps because the antioxidant systems were increased to compensate the potentially higher amounts of ROS. phages and production of pro-inflammatory mediators including TNF- $\alpha$ in the visceral adipose tissue (Marek et al, 2015).

Although the results indicate the precursor to IL-1 $\beta$ - pro-IL-1 $\beta$, was accumulated intracellularly, extracellular IL- $1 \beta$ remained undetected. We speculate that this might be due to the duration of incubation. A time course experiment in our research group ( $\mathrm{Su}-$ nasee et al, 2015) showed that pro-IL-1 $\beta$ accumulation is also time dependent. In the experiment using cellulose nanocrystals (CNCs) cationic derivatives, seven hours of treatment was the peak time 
for pro-IL-1 $\beta$ detection in J774A1 cells. Treatments at other time points did not seem to show as much pro-IL-1 $\beta$ accumulation. In the future, we would vary the lengths of treatments and investigate if this is a factor affecting pro- IL- $1 \beta$ accumulation and IL-1 $\beta$ secretion in fructose and glucose treatments. We also would like to investigate the effect of adding antioxidants in the current treatments and possibly, including lipids in treatments to create a more type 2-diabetic/metabolic-syndrome environment.

Future studies should focus on quantifying the cell counts and analyzing the MitoSox and JC-1 fluorescence imaging data statistically. Overall, our results corroborate with the existing information regarding the effects of excess sugar on ROS production by the mitochondria, and the potential link with activation of NLRP3 inflammasome pathway. By understanding the behavior of this pathway, researchers will be able to better understand autoimmune diseases and possibly create new ideas on mitochondria-targeted therapies.

\section{ACKNOWLEGEMENTS}

I am extremely grateful to my mentor, Dr. Karina Ckless, whose continuous guidance and encouragement made it possible to execute this study. In addition, I would like to thank the SUNY Plattsburgh Redcay Honors center for their approval and sponsorship of the project.

\section{REFERENCES}

Bray, G. A. (2004). Medical Consequences of Obesity. The Journal of Clinical Endocrinology \& Metabolism, 89(6), 2583-2589. http://doi.org/10.1210/ jc.2004-0535

Ceriello, A., \& Motz, E. (2004). Is Oxidative Stress the Pathogenic Mechanism Underlying Insulin Resistance, Diabetes, and Cardiovascular Disease? The Common Soil Hypothesis Revisited. Arteriosclerosis, Thrombosis, and Vascular Biology, 24(5), 816-823. http://doi.org/10.1161/01. ATV.0000122852.22604.78

Cha, S. H., Wolfgang, M., Tokutake, Y., Chohnan, S., \& Lane, M. D. (2008). Differential effects of central fructose and glucose on hypothalamic malonylCoA and food intake. Proceedings of the National Academy of Sciences of the United States of America, 105(44), 16871-16875. http://doi.org/10.1073/ pnas.0809255105

Devasagayam, T. P. A., Tilak, J. C., Boloor, K. K., Sane, K. S., Ghaskadbi, S. S., \& Lele, R. D. (2004). Free radicals and antioxidants in human health: Current status and future prospects. Journal of Association of Physicians of India, 52(OCT), 794-804.

Finucane, M. M., Stevens, G. A., Cowan, M. J., Danaei, G., Lin, J. K., Paciorek, C. J., Ezzati, M. (2011). National, regional, and global trends in body-mass index since 1980: systematic analysis of health examination surveys and epidemiological studies with 960 country-years and 9.1 million participants. The Lancet, 377(9765), 557-67.

Gao, D., Madi, M., Ding, C., Fok, M., Steele, T., Ford, C., ... Bing, C. (2014). Interleukin-1 $\beta$ mediates macrophage-induced impairment of insulin signaling in human primary adipocytes. American Journal of Physiology - Endocrinology and Metabolism, 307(3), E289-E304. http://doi.org/10.1152/ ajpendo.00430.2013

Gunter, M. J., \& Leitzmann, M. F. (2006). Obesity and colorectal cancer: epidemiology, mechanisms and candidate genes. The Journal of Nutritional Biochemistry, 17(3), 145-156. http://doi.org/10.1016/j.jnutbio.2005.06.011

Hajer, G. R., Haeften, T. W. van, \& Visseren, F. L. J. (2008). Adipose tissue dysfunction in obesity, diabetes, and vascular diseases. European Heart Journal, 29(24), 2959-2971. http://doi.org/10.1093/eurheartj/ehn387

Jo, E.-K., Kim, J. K., Shin, D.-M., \& Sasakawa, C. (2016). Molecular mechanisms regulating NLRP3 inflammasome activation. Cellular \& Molecular Immunology, 13(2), 148-159. http://doi.org/10.1038/cmi.2015.95

Larsen, C. M., Faulenbach, M., Vaag, A., Vølund, A., Ehses, J. A., Seifert, B., Do- nath, M. Y. (2007). Interleukin-1-Receptor Antagonist in Type 2 Diabetes Mellitus. The New England Journal of Medicine, 356(15), 1517-26. http:// doi.org/http://dx.doi.org/10.1056/NEJMoa065213

Lenaz, G. (2001). The Mitochondrial Production of Reactive Oxygen Species: Mechanisms and Implications in Human Pathology. IUBMB Life, 52(3-5), 159-164. http://doi.org/10.1080/15216540152845957

Liu, H., \& Heaney, A. P. (2011). Refined fructose and cancer. Expert Opinion on Therapeutic Targets, 15(9), 1049-1059. http://doi.org/10.1517/14728222.20 11.588208

Marek, G., Pannu, V., Shanmugham, P., Pancione, B., Mascia, D., Crosson, S., ... Sautin, Y. Y. (2015). Adiponectin Resistance and Proinflammatory Changes in the Visceral Adipose Tissue Induced by Fructose Consumption via Ketohexokinase-Dependent Pathway. Diabetes, 64(2), 508-518. http://doi. org/10.2337/db14-0411

Nguyen, T., Nioi, P., \& Pickett, C. B. (2009). The Nrf2-Antioxidant Response Element Signaling Pathway and Its Activation by Oxidative Stress. Journal of Biological Chemistry, 284(20), 13291-13295. http://doi.org/10.1074/jbc. R900010200

Nordberg, J., \& Arnér, E. S. J. (2001). Reactive oxygen species, antioxidants, and the mammalian thioredoxin system1. Free Radical Biology and Medicine, 31(11), 1287-1312. http://doi.org/10.1016/S0891-5849(01)00724-9

Popkin, B. M. (2001). The Nutrition Transition and Obesity in the Developing World. The Journal of Nutrition, 131(3), 871S-873S.

Reuter, S., Gupta, S. C., Chaturvedi, M. M., \& Aggarwal, B. B. (2010). Oxidative stress, inflammation, and cancer: How are they linked? Free Radical Biology and Medicine, 49(11), 1603-1616. http://doi.org/10.1016/j.freeradbiomed.2010.09.006

Rodriguez-Hernandez, H., Simental-Mendia, L. E., Ndez, H., Rodriguez-Ramirez, G., \& Reyes-Romero, M. A. (2013). Obesity and Inflammation: Epidemiology, Risk Factors, and Markers of Inflammation. International Journal of Endocrinology, 2013, e678159. http://doi.org/10.1155/2013/678159

Russell, J. W., Golovoy, D., Vincent, A. M., Mahendru, P., Olzmann, J. A., Mentzer, A., \& Feldman, E. L. (2002). High glucose-induced oxidative stress and mitochondrial dysfunction in neurons. The FASEB Journal, 16(13), 17381748. http://doi.org/10.1096/fj.01-1027com

Sunasee, R., Araoye, E., Pyram, D., Hemraz, U. D., Boluk, Y., \& Ckless, K. (2015). Cellulose nanocrystal cationic derivative induces NLRP3 inflammasomedependent IL-1 $\beta$ secretion associated with mitochondrial ROS production. Biochemistry and Biophysics Reports, 4, 1-9. http://doi.org/10.1016/j. bbrep.2015.08.008

Thannickal, V. J., \& Fanburg, B. L. (2000). Reactive oxygen species in cell signaling. American Journal of Physiology - Lung Cellular and Molecular Physiology, 279(6), L1005-L1028.

Tschopp, J., \& Schroder, K. (2010). NLRP3 inflammasome activation: The convergence of multiple signalling pathways on ROS production? Nature Reviews. Immunology, 10(3), 210-215. http://doi.org/10.1038/nri2725

Tsujimoto, Y., \& Shimizu, S. (2007). Role of the mitochondrial membrane permeability transition in cell death. Apoptosis: An International Journal On Programmed Cell Death, 12(5), 835-840. 